\title{
Dietary adequacy and dietary quality of Invit in the Canadian Arctic who smoke and the potential implications for chronic disease
}

\author{
Stacey E Rittmueller, Cindy Roache and Sangita Sharma* \\ Department of Medicine, University of Alberta, 1-126 Li Ka Shing Centre for Health Research Innovation, \\ Edmonton, Alberta T6G 2E1, Canada
}

Submitted 3 November 2010: Accepted 8 December 2011: First published online 24 January 2012

\begin{abstract}
Objective: To compare dietary intake and quality among adult Inuit by smoking status.

Design: A cross-sectional study using data from a validated quantitative FFQ.

Setting: Three isolated communities in Nunavut, Canada.

Subjects: Adult Inuit ( $n$ 208), aged between 19 and 79 years, from randomly selected households.

Results: Average energy intake did not differ between male smokers ( $n$ 22) and non-smokers ( $n$ 14; $16235 \mathrm{~kJ}$ and $13503 \mathrm{~kJ} ; P=0 \cdot 18$ ), but was higher among female smokers ( $n$ 126) compared with non-smokers ( $n$ 46; $12704 \mathrm{~kJ}$ and $8552 \mathrm{~kJ}$; $P<0 \cdot 0001)$. Average daily nutrient intakes were similar among men and higher among female smokers compared with non-smokers for all nutrients $(P \leq 0 \cdot 05)$ except $n$-3 fatty acids, vitamin A, vitamin D and Se. Female smokers had lower intake densities of thiamin, niacin, vitamin $\mathrm{B}_{6}$, folate, $\mathrm{Mg}, \mathrm{Na}(P \leq 0 \cdot 05)$, protein, $n-3$ fatty acids, cholesterol, Fe $(P \leq 0 \cdot 01)$, vitamin $\mathrm{B}_{12}$ and $\mathrm{Se}(P \leq 0 \cdot 001)$. Between $20 \%$ and $50 \%$ of male and female smokers were below the Dietary Reference Intake (DRI) for $\mathrm{Ca}$, folate, $\mathrm{Mg}$ and vitamins $\mathrm{A}$ and $\mathrm{K}$, and more than $50 \%$ were below the DRI for fibre and vitamin E. The proportion of smokers below the DRI was lower for all nutrients, except fibre and folate among men. Among smokers, non-nutrient-dense foods and traditional foods contributed less to energy $(-2 \cdot 1 \%$ and $-2 \cdot 0 \%$, respectively).

Conclusions: Adult smokers consumed fewer nutrient-dense, traditional foods, but had increased energy intake, which likely contributed to fewer dietary inadequacies compared with non-smokers. Promoting traditional food consumption supplemented with market-bought fruits and vegetables is important to improve dietary quality, especially among smokers.
\end{abstract}

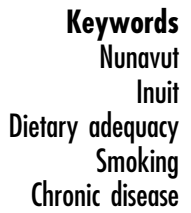

The burden of chronic disease is becoming increasingly apparent among Inuit in the Canadian Arctic. Compared with all of Canada, life expectancy is 13 years lower and stroke mortality is two times higher among Inuit ${ }^{(1)}$, and the prevalence of self-reported heart disease is three times higher among Aboriginal populations (i.e. First Nations, Metis and Inuit/Inuvialuit) ${ }^{(2)}$. Behavioural risk factors such as smoking and diet might play a central role in chronic disease development and mortality among Inuit $^{(3-5)}$. The effects of smoking on cardiovascular and respiratory diseases, stroke and certain cancers are well recognized ${ }^{(6,7)}$. Similarly, diets high in energy and fat and low in micronutrients have been linked to diseases such as obesity, CVD and cancer $^{(8)}$. Both diet and smoking induce physiological changes, such as increased endothelial damage, oxidized low-density lipoproteins and atherosclerosis, that increases risk for development of these diseases ${ }^{(9-11)}$. In addition to its direct effect on tissues, smoking can contribute to unbalanced nutrient profiles through a combination of altered taste preferences, metabolism and demand of certain nutrients, such as folate, $\beta$-carotene, Se, $\mathrm{Ca}$ and vitamin $\mathrm{C}^{(9,12,13)}$. Clustering of multiple behavioural risk factors such as smoking, low levels of physical activity, high alcohol consumption and poor diet, especially low intake of fruits and vegetables, may further increase the risk of chronic disease and the adversity of associated health outcomes. Diets of smokers in several populations have been shown to differ from those of non-smokers and have included more processed foods, meat and dairy and fewer fruits and vegetables ${ }^{(14)}$. Subsequently, higher intake of saturated fat and lower intakes of micronutrients 
such as vitamin $\mathrm{C}$, vitamin $\mathrm{A}$, folate and fibre have been reported among smokers ${ }^{(12,15)}$.

There is limited health research overall among Inuit and, to the authors' knowledge, specifically there are no previous studies that have explored dietary intake differences among Inuit exclusively by smoking status. Previous research among Inuit in Nunavut, Canada, highlighted inadequate intakes of dietary fibre, $\mathrm{Ca}$, folate and vitamins $\mathrm{A}, \mathrm{D}$ and $\mathrm{E}^{(5)}$. According to the Aboriginal Peoples Survey in 2006, smoking prevalence among Inuit in Nunavut was $64 \%$, and the highest prevalence was among residents $20-24$ years of age $(73 \%)^{(16)}$. The high prevalence of chronic disease and lower life expectancy among this population warrant the exploration of known chronic disease risk factors to create targeted disease prevention efforts. The purpose of the present study was to compare dietary intake and quality between adult Inuit smokers and non-smokers in Nunavut, Canada.

\section{Methods}

The survey instrument and data collection protocol have been described elsewhere ${ }^{(17,18)}$. In brief, a validated quantitative FFQ (QFFQ) designed specifically for this population was administered between June 2008 and October 2008 to Inuit adults in three isolated communities in Nunavut ${ }^{(17-19)}$. Households were randomly selected from three communities using housing maps provided by the local government. One individual per household was recruited to participate, namely the main food shopper and/or preparer, and these were typically women. Children ( $<19$ years) as well as pregnant and breast-feeding women were excluded due to their altered and changing dietary requirements. Participants reported the consumption amount and frequency of 150 items during a $30 \mathrm{~d}$ period by using three-dimensional food models and choosing from eight categories ranging from 'never' to 'two or more times per day ${ }^{(18)}$. A food composition table specific for this population was developed using the Canadian food composition tables within NutriBase, Clinical Nutrition Manager version $7 \cdot 17$ (CyberSoft Inc., Phoenix, AZ, USA), supplemented with data from the Canadian Nutrient File ${ }^{(20)}$.

The non-nutrient-dense foods (NNDF) category comprised the following foods: butter/margarine, jam/ marmalade, sugar/honey, non-dairy coffee whitener, hash browns/potato patties/French fries, gravy, salad dressing including mayonnaise and dips, ice cream, cakes/muffins, pies, cheesecake, chocolate bars, potato chips, crackers, cookies, hard candy, popcorn, granola bars, sweetened drinks and unsweetened drinks.

Traditional food items were defined as those obtained through subsistence practices, such as hunting and fishing, and were grouped into the following three categories: (i) traditional land foods (caribou, polar bear, musk ox and the organs of these animals); (ii) traditional sea foods (seal, seal liver, muktuk (whale skin and fat)) and various kinds of wild local fish (e.g. char and trout); and (iii) traditional sky foods (goose and ptarmigan).

The mean and standard deviation of daily energy and nutrient intakes were calculated for all participants. The energy intake requirement was defined using the Institute of Medicine's ${ }^{(21)}$ estimated amount of kilojoules needed to maintain energy balance for men or women aged 31-50 years at the very low physical activity-sedentary level because of previously documented low physical activity levels among this population ${ }^{(22,23)}$. Nutrients of interest were selected based on published effects of smoking on decreased antioxidant potential and dietary risk factors for chronic disease. To compare the nutrient intakes of smokers with non-smokers, nutrient densities per $4184 \mathrm{~kJ}$ (1000 kcal) were determined by dividing each participant's daily nutrient intakes by their energy intake (kcal), multiplied by 4184 . As nutrient densities were not normally distributed, the non-parametric Wilcoxon rank-sum test was used to determine differences in nutrient densities between smokers and non-smokers. Smokers were defined as consuming more than one cigarette daily. All smokers were treated the same, regardless of pack-years, due to small sample size.

Dietary adequacy was calculated using the Estimated Average Requirements (EAR) based on the gender- and agespecific (19-30 years, 31-50 years, $51-70$ years, $>70$ years) recommendations ${ }^{(24)}$. Where the EAR was not available, as for dietary fibre, vitamin $\mathrm{D}$, vitamin $\mathrm{K}$, pantothenic acid, $\mathrm{K}$, $\mathrm{Na}$ and $\mathrm{Ca}$, the Adequate Intake (AI) was used, as recommended $^{(21)}$. Due to the small sample size per age group, the number and percentage of participants not meeting the recommendations for selected nutrients were first determined by age-specific EAR and then pooled for all age groups.

Participants who reported extreme energy intake $>20920 \mathrm{~kJ}(>5000 \mathrm{kcal} ; n$ 3) were excluded from the analysis. No participant reported an energy intake of $<2092 \mathrm{~kJ}(<500 \mathrm{kcal})$. All analyses were stratified by gender and smoking status. Data were analysed using the SAS statistical software package version 9.2 (SAS Institute, Inc., Cary, NC, USA). All tests and $P$ values were twosided and considered statistically significant at $\alpha=0 \cdot 05$. Institutional Review Board approval was obtained from the Committee on Human Studies at the University of Hawaii and the Office of Human Research Ethics at the University of North Carolina at Chapel Hill. The Nunavut Research Institute licensed the present study.

\section{Results}

The prevalence of smoking among men and women in the present study was $61 \%$ and $73 \%$, respectively. Men who reported smoking ( $n$ 22) consumed on average $16235 \mathrm{~kJ}$ (3880 kcal) daily, which is $2732 \mathrm{~kJ}$ (653 kcal) more than men who did not smoke ( $n$ 14) and $7030 \mathrm{~kJ}$ 
Table 1 Daily intakes of energy and selected nutrients among adult Inuit in Nunavut, Canada, by gender and smoking status, 2008

\begin{tabular}{|c|c|c|c|c|c|c|c|c|c|c|}
\hline \multirow[b]{3}{*}{ Nutrient } & \multicolumn{5}{|c|}{ Men } & \multicolumn{5}{|c|}{ Women } \\
\hline & \multicolumn{2}{|c|}{$\begin{array}{l}\text { Non-smoker } \\
\quad(n \text { 14) }\end{array}$} & \multicolumn{2}{|c|}{$\begin{array}{l}\text { Smoker } \\
(n \text { 22) }\end{array}$} & \multirow[b]{2}{*}{ DRIt } & \multicolumn{2}{|c|}{$\begin{array}{l}\text { Non-smoker } \\
\quad(n \text { 46) }\end{array}$} & \multicolumn{2}{|c|}{$\begin{array}{l}\text { Smoker } \\
(n 126)\end{array}$} & \multirow[b]{2}{*}{ DRIt } \\
\hline & Mean & $S D$ & Mean & SD & & Mean & SD & Mean & SD & \\
\hline Age (years) & $47 \cdot 7$ & $16 \cdot 8$ & 38.5 & $12 \cdot 9$ & - & $52 \cdot 4$ & $14 \cdot 4$ & $38 \cdot 7^{\star \star *}$ & $10 \cdot 7$ & - \\
\hline Energy $(\mathrm{kJ}) \ddagger, \S$ & 13503 & 6069 & 16235 & 6081 & 9205 & 8552 & 3634 & $12704^{\star \star}$ & 5319 & 7531 \\
\hline$\% \mathrm{E}$ from protein $\|$ & 20 & $7 \cdot 3$ & 21 & $6 \cdot 1$ & $10-35$ & 22 & $5 \cdot 5$ & 21 & $7 \cdot 2$ & $10-35$ \\
\hline$\% \mathrm{E}$ from carbohydrates $\|$ & 45 & $8 \cdot 6$ & 44 & $9 \cdot 3$ & $45-65$ & 43 & $9 \cdot 4$ & 45 & 8.8 & $45-65$ \\
\hline$\% \mathrm{E}$ from fat $\|$ & 29 & $5 \cdot 2$ & 31 & $5 \cdot 6$ & $20-35$ & 26 & $5 \cdot 2$ & 27 & $5 \cdot 7$ & $20-35$ \\
\hline Protein $(\mathrm{g})$ & 174 & $83 \cdot \overline{8}$ & 209 & $96 \cdot 8$ & - & 130 & $54 \cdot 9$ & $175^{\star \star}$ & $106 \cdot 0$ & - \\
\hline Carbohydrate (g) & 388 & $221 \cdot 7$ & 442 & $162 \cdot 9$ & - & 236 & $122 \cdot 1$ & $358^{* * *}$ & $173 \cdot 5$ & - \\
\hline Sugars $(g)$ & 187 & $159 \cdot 7$ & 232 & $115 \cdot 0$ & $<25 \% \mathrm{E}$ & 112 & 73.9 & $179^{\star \star \star}$ & $123 \cdot 7$ & $<25 \% \mathrm{E}$ \\
\hline Dietary fibre (g) & $15 \cdot 8$ & $7 \cdot 8$ & $19 \cdot 7$ & $9 \cdot 0$ & 38 & $10 \cdot 9$ & $5 \cdot 9$ & $14 \cdot 6^{\star \star \star}$ & $6 \cdot 7$ & 25 \\
\hline Fat (g) & $104 \cdot 0$ & $42 \cdot 7$ & $137 \cdot 1$ & 63.9 & - & $63 \cdot 0$ & $27 \cdot 2$ & $95 \cdot 8^{\star \star \star}$ & $41 \cdot 8$ & - \\
\hline SFAtt (g) & 35.9 & $16 \cdot 4$ & $48 \cdot 2$ & $24 \cdot 0$ & $<10 \% \mathrm{E}$ & $20 \cdot 7$ & $9 \cdot \overline{8}$ & $33 \cdot 9^{\star \star \star}$ & $14 \cdot 7$ & $<10 \% \mathrm{E}$ \\
\hline MUFA (g) & $36 \cdot 0$ & $14 \cdot 6$ & 48.8 & $22 \cdot 6$ & - & $22 \cdot 3$ & $9 \cdot 9$ & $32 \cdot 8^{\star \star \star}$ & $14 \cdot 3$ & - \\
\hline PUFA (g) & $16 \cdot 3$ & $7 \cdot 0$ & $20 \cdot 2$ & $9 \cdot 4$ & - & $10 \cdot 3$ & 4.9 & $14 \cdot 5^{\star \star \star}$ & $6 \cdot 6$ & - \\
\hline$n-3$ fatty acids (g) & $2 \cdot 1$ & $1 \cdot 4$ & $2 \cdot 1$ & $1 \cdot 4$ & - & $1 \cdot 4$ & 0.7 & $1 \cdot 7$ & 1.3 & - \\
\hline$n-6$ fatty acids (g) & $12 \cdot 0$ & $6 \cdot 2$ & $15 \cdot 8$ & $8 \cdot 2$ & - & $7 \cdot 0$ & $4 \cdot 4$ & $10 \cdot 6^{\star \star \star}$ & $5 \cdot 0$ & - \\
\hline Cholesterol (mg) & 530 & $273 \cdot 7$ & 730 & $458 \cdot 1$ & As low as possible & 428 & $412 \cdot 1$ & $491^{\star \star *}$ & 308.9 & As low as possible \\
\hline Vitamin Ał‡ ( $\mu g$-RAE) & 831 & $599 \cdot 5$ & 893 & $485 \cdot 5$ & 900 & 842 & $839 \cdot 7$ & $1155^{\star \star \star}$ & 1632 & 700 \\
\hline Thiaminł‡ (mg) & $2 \cdot 4$ & 0.9 & $2 \cdot 9$ & $1 \cdot 1$ & $1 \cdot 2$ & $1 \cdot 8$ & 0.7 & $2 \cdot 3^{\star \star \star}$ & $1 \cdot 1$ & $1 \cdot 1$ \\
\hline Riboflavin $\neq$ (mg) & $4 \cdot 1$ & $1 \cdot 4$ & $5 \cdot 2$ & $2 \cdot 4$ & $1 \cdot 3$ & $3 \cdot 1$ & $1 \cdot 8$ & $4 \cdot 2^{\star \star \star}$ & $2 \cdot 1$ & $1 \cdot 1$ \\
\hline Niacinłł (mg) & $40 \cdot 6$ & 21.5 & 51.8 & $26 \cdot 8$ & 16 & $27 \cdot 2$ & $11 \cdot 7$ & $36 \cdot 9^{\star \star \star}$ & $18 \cdot 4$ & 14 \\
\hline Pantothenic acid $(\mathrm{mg})$ & $9 \cdot 6$ & $4 \cdot 1$ & $12 \cdot 7$ & $5 \cdot 9$ & 5 & $6 \cdot 9$ & 3.5 & $10 \cdot 4^{\star \star \star}$ & $6 \cdot 1$ & 5 \\
\hline Vitamin $B_{6} \neq \neq(m g)$ & $2 \cdot 3$ & $1 \cdot 3$ & $3 \cdot 1$ & $1 \cdot 4$ & $1 \cdot 3$ & 1.6 & $0 \cdot 8$ & $2 \cdot 1^{\star \star \star}$ & $1 \cdot 0$ & $1 \cdot 3$ \\
\hline Total folate $¥$ ( $\mu$ g-DFE) & 445 & $143 \cdot 7$ & 505 & $194 \cdot 3$ & 400 & 315 & $141 \cdot 8$ & $415^{\star \star \star}$ & $177 \cdot 8$ & 400 \\
\hline Vitamin $B_{12} \neq \ddagger(\mu \mathrm{g})$ & $16 \cdot 2$ & $9 \cdot 4$ & $17 \cdot 6$ & $13 \cdot 8$ & $2 \cdot 4$ & $16 \cdot 3$ & $14 \cdot 2$ & $16 \cdot 5$ & $15 \cdot 2$ & $2 \cdot 4$ \\
\hline Feł‡ (mg) & $30 \cdot 8$ & $13 \cdot 3$ & $32 \cdot 2$ & $12 \cdot 7$ & 8 & $23 \cdot 0$ & $11 \cdot 2$ & $29 \cdot 8^{\star}$ & $18 \cdot 2$ & 18 \\
\hline 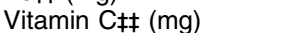 & 193 & $222 \cdot 4$ & 215 & $115 \cdot 2$ & 90 & 132 & $106 \cdot 7$ & $202^{\star \star \star}$ & $135 \cdot 5$ & 75 \\
\hline Vitamin D,$\S \S(\mu \mathrm{g})$ & $5 \cdot 1$ & $3 \cdot 0$ & $8 \cdot 8$ & $7 \cdot 2$ & 5 & $4 \cdot 1$ & $3 \cdot 3$ & $5 \cdot 3$ & 4.9 & 5 \\
\hline 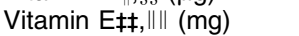 & $4 \cdot 6$ & $2 \cdot 0$ & $5 \cdot 8$ & $2 \cdot 9$ & 15 & 3.0 & $1 \cdot 6$ & $4 \cdot 3^{\star \star \star}$ & $2 \cdot 6$ & 15 \\
\hline Vitamin $K^{\top}(\mu \mathrm{g})$ & $113 \cdot 4$ & $94 \cdot 6$ & $164 \cdot 0$ & $135 \cdot 3$ & 120 & 53.9 & $43 \cdot 2$ & $132 \cdot 0^{\star \star \star}$ & 114.9 & 90 \\
\hline $\mathrm{Ca} \sigma^{(m g)}$ & 1060 & $635 \cdot 7$ & 1412 & $670 \cdot 3$ & 1000 & 802 & $557 \cdot 2$ & $1223^{\star \star \star}$ & 639.5 & 1000 \\
\hline 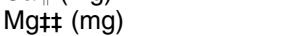 & 411 & $178 \cdot 0$ & 471 & $164 \cdot 2$ & 420 & 293 & $106 \cdot 4$ & $396^{\star \star \star}$ & $164 \cdot 8$ & 320 \\
\hline $\mathrm{K}^{\top}(\mathrm{g})$ & $4 \cdot 5$ & $2 \cdot 0$ & $5 \cdot 2$ & $2 \cdot 1$ & $4 \cdot 7$ & $3 \cdot 2$ & $1 \cdot 3$ & $4 \cdot 4^{\star \star \star}$ & $2 \cdot 0$ & $4 \cdot 7$ \\
\hline $\mathrm{Na}$ ( $(\mathrm{g})$ & $4 \cdot 7$ & $1 \cdot 8$ & $5 \cdot 3$ & $2 \cdot 3$ & 1.5 & $3 \cdot 1$ & $1 \cdot 3$ & $4 \cdot 3^{\star \star \star}$ & $2 \cdot 4$ & 1.5 \\
\hline Seft $(\mu \mathrm{g})$ & 152 & $79 \cdot 5$ & 198 & $97 \cdot 6$ & 55 & 150 & $94 \cdot 7$ & 176 & $208 \cdot 1$ & 55 \\
\hline Znł‡ (mg) & $19 \cdot 9$ & $8 \cdot 4$ & $24 \cdot 7$ & $10 \cdot 6$ & 11 & $15 \cdot 4$ & $7 \cdot 5$ & $21 \cdot 7^{\star \star \star}$ & $14 \cdot 2$ & 8 \\
\hline
\end{tabular}

DRI, Dietary Reference Intake; \%E, percentage of energy; RAE, retinol activity equivalents; DFE, dietary folate equivalents.

Mean values were significantly different from those of non-smokers of the same gender: ${ }^{\star} P \leq 0 \cdot 05,{ }^{\star \star} P \leq 0 \cdot 01,{ }^{* \star} P \leq 0 \cdot 001$.
tThe DRI are presented using Adequate Intake/RDA for men and women aged $31-50$ years, Acceptable Macronutrient Distribution Ranges and the recommendation on saturated fat intake by Joint WHO/FAO ${ }^{(21,46)}$. $+1 \mathrm{kcal}=4 \cdot 184 \mathrm{~kJ}$.
$\$$ Estimated kilojoules needed to maintain energy balance for men or women aged $31-50$ years at the very low physical activity-sedentary level. \$Estimated kilojoules needed to maintain energy
$\|$ Acceptable Macronutrient Distribution Range.

$\|$ Acceptable Macronutrient Distribution Rang

Adequate Intake.

on saturated fat intake by Joint WHO/FAO ${ }^{(46)}$

$\$ \S A$ s cholecalciferol in the absence of adequate exposure to sunlight.

\|\| As $\alpha$-tocopherol. 
(1680 kcal) above the Dietary Reference Intake (DRI) for energy ( 9205 kJ/2200 kcal daily). Female smokers ( $n$ 126) consumed on average $12704 \mathrm{~kJ}(3036 \mathrm{kcal})$ daily compared with non-smoking women ( $n$ 46) who consumed an average of $8552 \mathrm{~kJ}$ (2043 kcal) daily. Daily energy intake was $5173 \mathrm{~kJ}(1236 \mathrm{kcal})$ and $1021 \mathrm{~kJ}(243 \mathrm{kcal})$ above the DRI $(7531 \mathrm{~kJ} / 1800 \mathrm{kcal})$ for female smokers and non-smokers, respectively. The mean (SD) number of cigarettes smoked daily among male and female smokers was $8 \cdot 9(6 \cdot 2)$ and $8 \cdot 0(4 \cdot 9)$, respectively (data not shown). Average daily energy and nutrient intakes did not differ between male smokers and non-smokers, and all men regardless of smoking status had average intakes below the DRI for fibre, vitamin A and vitamin E (Table 1). Average daily energy and nutrient intakes were higher among female smokers compared with non-smokers for all nutrients $(P<0 \cdot 05)$ except $n-3$ fatty acids, vitamin A, vitamin $\mathrm{D}$ and Se. Average intakes of fibre, $\mathrm{K}$ and vitamin $\mathrm{E}$ were below recommendations for all females.

The majority of participants were below the DRI for dietary fibre (90-100\%) and vitamin E (90-100\%), regardless of smoking status or gender. Between $20 \%$ and $50 \%$ of male smokers were below the DRI for Ca, folate, $\mathrm{Mg}, \mathrm{K}$ and vitamins $\mathrm{A}, \mathrm{D}$ and $\mathrm{K}$, and more than $50 \%$ were below the DRI for fibre and vitamin E; however, the proportion of male smokers below the DRI for these nutrients was lower compared with non-smokers, with the exception of folate (Table 2). Considering female smokers, between $20 \%$ and $50 \%$ were below the DRI for $\mathrm{Ca}$, folate, $\mathrm{Mg}$ and vitamins $\mathrm{A}$ and $\mathrm{K}$, and more than $50 \%$ were below the DRI for fibre, $\mathrm{K}$ and vitamins $\mathrm{D}$ and $\mathrm{E}$, although these proportions were lower compared with non-smoking females (Table 2).

No significant differences were seen in nutrient intake density (per $4184 \mathrm{~kJ} / 1000 \mathrm{kcal}$ ) between male smokers and non-smokers (Table 3). Female smokers had significantly lower intake densities of thiamin, niacin, vitamin $\mathrm{B}_{6}$, folate, $\mathrm{Mg}, \mathrm{Na}(P \leq 0 \cdot 05)$, protein, $n-3$ fatty acids, cholesterol, Fe $(P \leq 0 \cdot 01)$, vitamin $\mathrm{B}_{12}$ and $\mathrm{Se}(P \leq 0 \cdot 001)$, and significantly higher intake densities of saturated fat and vitamin $\mathrm{K}$ $(P \leq 0 \cdot 01$; Table 3$)$.

Among men and women combined, the primary contributors to energy, protein and carbohydrates were the same regardless of smoking status (Table 4). Comparing smokers with non-smokers, NNDF contributed less to energy $(-2 \cdot 1 \%)$, fat $(-4 \cdot 3 \%)$ and carbohydrates $(-1 \cdot 3 \%)$. The contribution of traditional foods to energy was lower $(-2 \cdot 0 \%)$ among adults who smoked compared with nonsmokers. Smokers had similar contributions of dairy to energy $(6 \cdot 9 \% v \cdot 6 \cdot 3 \%)$ and fruits to energy $(3 \cdot 3 \% v \cdot 3 \cdot 7 \%)$.

\section{Discussion}

The present study supplements the limited literature available on nutrition of adult Inuit by exploring dietary

Table 2 Percentage of adult Inuit below the Dietary Reference Intakes by gender and smoking status, Nunavut, Canada, 2008

\begin{tabular}{|c|c|c|c|c|}
\hline \multirow[b]{3}{*}{ Nutrient } & \multicolumn{2}{|c|}{ Men } & \multicolumn{2}{|c|}{ Women } \\
\hline & $\begin{array}{c}\text { Non-smokers } \\
(n 14)\end{array}$ & $\begin{array}{c}\text { Smokers } \\
(n 22)\end{array}$ & $\begin{array}{c}\text { Non-smokers } \\
\quad(n \text { 46) }\end{array}$ & $\begin{array}{c}\text { Smokers } \\
(n \text { 126) }\end{array}$ \\
\hline & $\%$ & $\%$ & $\%$ & $\%$ \\
\hline Dietary fibre $(\mathrm{g}) \dagger$ & $100 \cdot 0$ & $100 \cdot 0$ & $95 \cdot 7$ & $91 \cdot 3$ \\
\hline $\mathrm{Ca}(\mathrm{mg}) \dagger$ & $57 \cdot 1$ & $27 \cdot 3$ & $78 \cdot 3$ & $43 \cdot 7$ \\
\hline Total folate $(\mu \mathrm{g}$-DFE) $\ddagger$ & $14 \cdot 3$ & $27 \cdot 3$ & $56 \cdot 5$ & $27 \cdot 8$ \\
\hline Vitamin A $(\mu \mathrm{g}-\mathrm{RAE}) \ddagger$ & $42 \cdot 9$ & $36 \cdot 4$ & $37 \cdot 0$ & $19 \cdot 8$ \\
\hline Vitamin $B_{6}(\mathrm{mg}) \ddagger$ & $7 \cdot 1$ & $0 \cdot 0$ & $32 \cdot 6$ & $13 \cdot 5$ \\
\hline Vitamin C (mg) $\ddagger$ & $28 \cdot 6$ & $4 \cdot 5$ & $34 \cdot 8$ & $13 \cdot 5$ \\
\hline Vitamin D $(\mu \mathrm{g})+, \S$ & $71 \cdot 4$ & $31 \cdot 8$ & $82 \cdot 6$ & $64 \cdot 3$ \\
\hline Vitamin E (mg)‡,\| & $100 \cdot 0$ & $95 \cdot 5$ & $100 \cdot 0$ & $97 \cdot 6$ \\
\hline $\mathrm{Fe}(\mathrm{mg}) \ddagger$ & $0 \cdot 0$ & 0.0 & $4 \cdot 3$ & $4 \cdot 0$ \\
\hline $\mathrm{Zn}(\mathrm{mg}) \ddagger$ & $14 \cdot 3$ & $4 \cdot 5$ & $13 \cdot 0$ & $6 \cdot 3$ \\
\hline Thiamin (mg)‡ & $0 \cdot 0$ & $0 \cdot 0$ & $4 \cdot 3$ & $6 \cdot 3$ \\
\hline Riboflavin (mg)‡ & $0 \cdot 0$ & $0 \cdot 0$ & $0 \cdot 0$ & $0 \cdot 0$ \\
\hline Niacin (mg) $\ddagger$ & $0 \cdot 0$ & $0 \cdot 0$ & $2 \cdot 2$ & $2 \cdot 4$ \\
\hline Pantothenic acid (mg)† & $14 \cdot 3$ & $9 \cdot 1$ & $32 \cdot 6$ & $16 \cdot 7$ \\
\hline Vitamin $B_{12}(\mu \mathrm{g}) \ddagger$ & 0.0 & $0 \cdot 0$ & $2 \cdot 2$ & $2 \cdot 4$ \\
\hline Vitamin $K(\mu \mathrm{g}) \dagger$ & $64 \cdot 3$ & $50 \cdot 0$ & $80 \cdot 4$ & $46 \cdot 0$ \\
\hline$M g(m g) \ddagger$ & $35 \cdot 7$ & $31 \cdot 8$ & $43 \cdot 5$ & $19 \cdot 8$ \\
\hline$K(g) t$ & $64 \cdot 3$ & $45 \cdot 5$ & $84 \cdot 8$ & $63 \cdot 5$ \\
\hline $\mathrm{Na}(\mathrm{g}) \dagger$ & $0 \cdot 0$ & $0 \cdot 0$ & $8 \cdot 7$ & $4 \cdot 8$ \\
\hline Se $(\mu \mathrm{g}) \ddagger$ & $0 \cdot 0$ & $0 \cdot 0$ & $4 \cdot 3$ & $4 \cdot 0$ \\
\hline
\end{tabular}

DFE, dietary folate equivalents; RAE, retinol activity equivalents.

tAdequate Intake used for comparison.

‡Estimated Average Requirement used for comparison.

$\S$ As cholecalciferol in the absence of adequate exposure to sunlight.

$\|$ As $\alpha$-tocopherol. 
Table 3 Nutrient density per $4184 \mathrm{~kJ}(1000 \mathrm{kcal})$ among adult Inuit by gender and smoking status, Nunavut, Canada, 2008

\begin{tabular}{|c|c|c|c|c|c|c|c|c|}
\hline \multirow[b]{3}{*}{ Nutrient } & \multicolumn{4}{|c|}{ Men } & \multicolumn{4}{|c|}{ Women } \\
\hline & \multicolumn{2}{|c|}{$\begin{array}{c}\text { Non-smokers } \\
(n 14)\end{array}$} & \multicolumn{2}{|c|}{$\begin{array}{c}\text { Smokers } \\
(n 22)\end{array}$} & \multicolumn{2}{|c|}{$\begin{array}{c}\text { Non-smokers } \\
(n 46)\end{array}$} & \multicolumn{2}{|c|}{$\begin{array}{c}\text { Smokers } \\
(n \text { 126) }\end{array}$} \\
\hline & Mean & SD & Mean & SD & Mean & SD & Mean & SD \\
\hline Protein $(\mathrm{g})$ & $54 \cdot 6$ & $18 \cdot 5$ & $53 \cdot 8$ & $16 \cdot 9$ & $65 \cdot 7$ & $19 \cdot 8$ & $56 \cdot 7^{\star \star}$ & $19 \cdot 9$ \\
\hline Carbohydrates (g) & 117 & $22 \cdot 1$ & 116 & $25 \cdot 0$ & 113 & $24 \cdot 9$ & 118 & $23 \cdot 6$ \\
\hline Sugars (g) & $52 \cdot 7$ & $21 \cdot 7$ & $60 \cdot 9$ & $26 \cdot 6$ & $51 \cdot 9$ & $25 \cdot 7$ & $58 \cdot 3$ & $24 \cdot 0$ \\
\hline Dietary fibre (g) & $5 \cdot 0$ & $1 \cdot 4$ & $5 \cdot 2$ & $1 \cdot 8$ & $5 \cdot 3$ & $1 \cdot 6$ & $5 \cdot 0$ & $1 \cdot 8$ \\
\hline Fat $(\mathrm{g})$ & $32 \cdot 9$ & $5 \cdot 6$ & $34 \cdot 8$ & $6 \cdot 0$ & $31 \cdot 1$ & $5 \cdot 1$ & $31 \cdot 8$ & $5 \cdot 7$ \\
\hline SFA (g) & $11 \cdot 4$ & $3 \cdot 0$ & $12 \cdot 2$ & $2 \cdot 6$ & $10 \cdot 1$ & $2 \cdot 3$ & $11 \cdot 3^{\star \star}$ & $2 \cdot 7$ \\
\hline MUFA (g) & $11 \cdot 4$ & $2 \cdot 2$ & $12 \cdot 4$ & $2 \cdot 4$ & $11 \cdot 1$ & $2 \cdot 3$ & $10 \cdot 9$ & $2 \cdot 3$ \\
\hline PUFA (g) & $5 \cdot 2$ & $1 \cdot 3$ & $5 \cdot 2$ & $1 \cdot 3$ & $5 \cdot 1$ & $1 \cdot 5$ & $4 \cdot 8$ & $1 \cdot 2$ \\
\hline$n-3$ fatty acids (g) & $0 \cdot 7$ & $0 \cdot 4$ & 0.5 & $0 \cdot 3$ & $0 \cdot 7$ & $0 \cdot 4$ & $0 \cdot 6^{\star *}$ & $0 \cdot 4$ \\
\hline$n-6$ fatty acids $(\mathrm{g})$ & $3 \cdot 8$ & 1.5 & $4 \cdot 0$ & $1 \cdot 2$ & $3 \cdot 3$ & $1 \cdot 6$ & $3 \cdot 6$ & $1 \cdot 2$ \\
\hline Cholesterol (mg) & 179 & $103 \cdot 9$ & 193 & $116 \cdot 0$ & 206 & $130 \cdot 1$ & $165^{\star \star}$ & $95 \cdot 0$ \\
\hline Vitamin A ( $\mu \mathrm{g}-\mathrm{RAE})$ & 275 & $220 \cdot 0$ & 233 & $98 \cdot 4$ & 434 & $550 \cdot 4$ & 396 & $574 \cdot 2$ \\
\hline Thiamin (mg) & $0 \cdot 8$ & 0.2 & $0 \cdot 8$ & $0 \cdot 2$ & 0.9 & 0.3 & $0 \cdot 8^{*}$ & 0.2 \\
\hline Riboflavin (mg) & $1 \cdot 4$ & $0 \cdot 4$ & $1 \cdot 4$ & 0.5 & 1.5 & 0.5 & $1 \cdot 4$ & $0 \cdot 6$ \\
\hline Niacin (mg) & $12 \cdot 5$ & $3 \cdot 2$ & $13 \cdot 1$ & $4 \cdot 0$ & $14 \cdot 0$ & $4 \cdot 7$ & $12 \cdot 5^{\star}$ & $4 \cdot 4$ \\
\hline Pantothenic acid (mg) & $3 \cdot 1$ & $1 \cdot 0$ & $3 \cdot 3$ & $1 \cdot 2$ & $3 \cdot 4$ & $1 \cdot 2$ & $3 \cdot 5$ & $1 \cdot 6$ \\
\hline Vitamin $\mathrm{B}_{6}(\mathrm{mg})$ & $0 \cdot 7$ & 0.2 & 0.8 & 0.2 & 0.8 & 0.2 & $0 \cdot 7^{*}$ & 0.2 \\
\hline Total folate ( $\mu \mathrm{g}$-DFE) & 147 & $35 \cdot 9$ & 133 & 34.5 & 160 & $44 \cdot 3$ & $144^{*}$ & $49 \cdot 5$ \\
\hline Vitamin $B_{12}(\mu \mathrm{g})$ & $5 \cdot 5$ & 3.5 & $4 \cdot 8$ & $4 \cdot 3$ & $8 \cdot 1$ & $5 \cdot 6$ & $5 \cdot 3^{\star \star \star}$ & $4 \cdot 0$ \\
\hline $\mathrm{Fe}(\mathrm{mg})$ & $10 \cdot 3$ & $4 \cdot 8$ & $8 \cdot 7$ & $3 \cdot 3$ & $11 \cdot 5$ & $3 \cdot 8$ & $9 \cdot 7^{\star \star}$ & $3 \cdot 9$ \\
\hline Vitamin C (mg) & $50 \cdot 9$ & $33 \cdot 0$ & $59 \cdot 3$ & $33 \cdot 1$ & $62 \cdot 4$ & $44 \cdot 6$ & $67 \cdot 3$ & $41 \cdot 2$ \\
\hline Vitamin D $(\mu \mathrm{g}) \dagger$ & $1 \cdot 8$ & $1 \cdot 3$ & $2 \cdot 3$ & $1 \cdot 8$ & $2 \cdot 1$ & 1.5 & $1 \cdot 8$ & $1 \cdot 4$ \\
\hline Vitamin E (mg)‡ & $1 \cdot 5$ & 0.5 & $1 \cdot 5$ & $0 \cdot 7$ & $1 \cdot 4$ & 0.5 & $1 \cdot 5$ & $0 \cdot 7$ \\
\hline Vitamin $\mathrm{K}(\mu \mathrm{g})$ & $37 \cdot 4$ & $30 \cdot 0$ & $41 \cdot 9$ & $26 \cdot 9$ & $27 \cdot 3$ & $20 \cdot 8$ & $47 \cdot 8^{\star \star}$ & $46 \cdot 4$ \\
\hline $\mathrm{Ca}(\mathrm{mg})$ & 347 & $160 \cdot 2$ & 369 & $146 \cdot 2$ & 389 & $180 \cdot 8$ & 417 & $198 \cdot 2$ \\
\hline $\mathrm{Mg}(\mathrm{mg})$ & 129 & $28 \cdot 3$ & 124 & $27 \cdot 5$ & 150 & $35 \cdot 3$ & $135^{*}$ & $30 \cdot 6$ \\
\hline $\mathrm{K}(\mathrm{g})$ & $1 \cdot 4$ & $0 \cdot 3$ & $1 \cdot 3$ & $0 \cdot 3$ & $1 \cdot 6$ & $0 \cdot 4$ & 1.5 & $0 \cdot 4$ \\
\hline $\mathrm{Na}(\mathrm{g})$ & 1.5 & $0 \cdot 3$ & $1 \cdot 4$ & $0 \cdot 3$ & $1 \cdot 5$ & $0 \cdot 4$ & $1 \cdot 4^{*}$ & $0 \cdot 4$ \\
\hline Se $(\mu \mathrm{g})$ & $47 \cdot 7$ & $17 \cdot 3$ & $50 \cdot 4$ & $15 \cdot 3$ & $74 \cdot 4$ & $36 \cdot 7$ & $55 \cdot 9^{\star \star \star}$ & $40 \cdot 0$ \\
\hline $\mathrm{Zn}(\mathrm{mg})$ & $6 \cdot 4$ & $2 \cdot 2$ & $6 \cdot 6$ & $2 \cdot 5$ & $7 \cdot 6$ & $2 \cdot 2$ & $7 \cdot 0$ & $2 \cdot 8$ \\
\hline
\end{tabular}

RAE, retinol activity equivalents; DFE, dietary folate equivalents.

Mean values were significantly different from those of non-smokers of the same gender: ${ }^{\star} P \leq 0 \cdot 05,{ }^{\star \star} P \leq 0 \cdot 01,{ }^{\star \star \star} P \leq 0 \cdot 001$.

tAs cholecalciferol in the absence of adequate exposure to sunlight.

$\ddagger$ As $\alpha$-tocopherol.

intake differences by smoking status. The smoking prevalence among men and women in our study was consistent with a recent Statistics Canada survey ${ }^{(16)}$. Overall, the comparison of dietary intake and quality by smoking status varied by gender. There were no significant differences between male smokers and nonsmokers in average daily energy and nutrient intakes or dietary quality; however, nutrient intakes were significantly higher and diet quality significantly lower for a majority of nutrients comparing female smokers and non-smokers. The energy intake among all participants was high; however, this was anticipated because this population is known to have high energy requirements due to the challenging climate. The QFFQ contains a large number of food items that make a substantial contribution to energy intake ${ }^{(19)}$. Despite the possible overestimation of energy intake, the QFFQ used in the present study was developed specifically for this population and has been shown to be a valid measure of dietary intake. As discussed in the Methods section, extreme intakes $(<2092 \mathrm{~kJ} /<500 \mathrm{kcal}$ and $>20920 \mathrm{~kJ} />5000 \mathrm{kcal})$ were excluded. The high energy intake levels are also reflected by high rates of overweight and obesity in this population $^{(5,18)}$.

A lower proportion of smokers did not meet the recommendations for all nutrients of interest, with the exception of fibre and folate among males. Therefore, non-smokers appeared to have fewer dietary inadequacies than smokers. Smokers had higher energy intake but consumed fewer nutrient-dense, traditional foods which might have contributed to inadequate nutrient intakes. The number of smokers with nutrient intakes below recommendations may not be very different from non-smokers in this population group; however, it is worth noting the increased demand tobacco and its metabolites places on certain nutrients, especially antioxidants such as vitamin C, vitamin $\mathrm{A}$ and $\beta$-carotene ${ }^{(13)}$.

Decreased antioxidant intake, particularly vitamins A and $\mathrm{C}^{(13)}$, have been documented among smokers in other populations and high levels of smoking-associated oxidative stress subsequently increase the antioxidant demand ${ }^{(25)}$. Contradicting these observations from previous literature, smokers in the present study had higher average daily intakes and similar nutrient densities compared with 


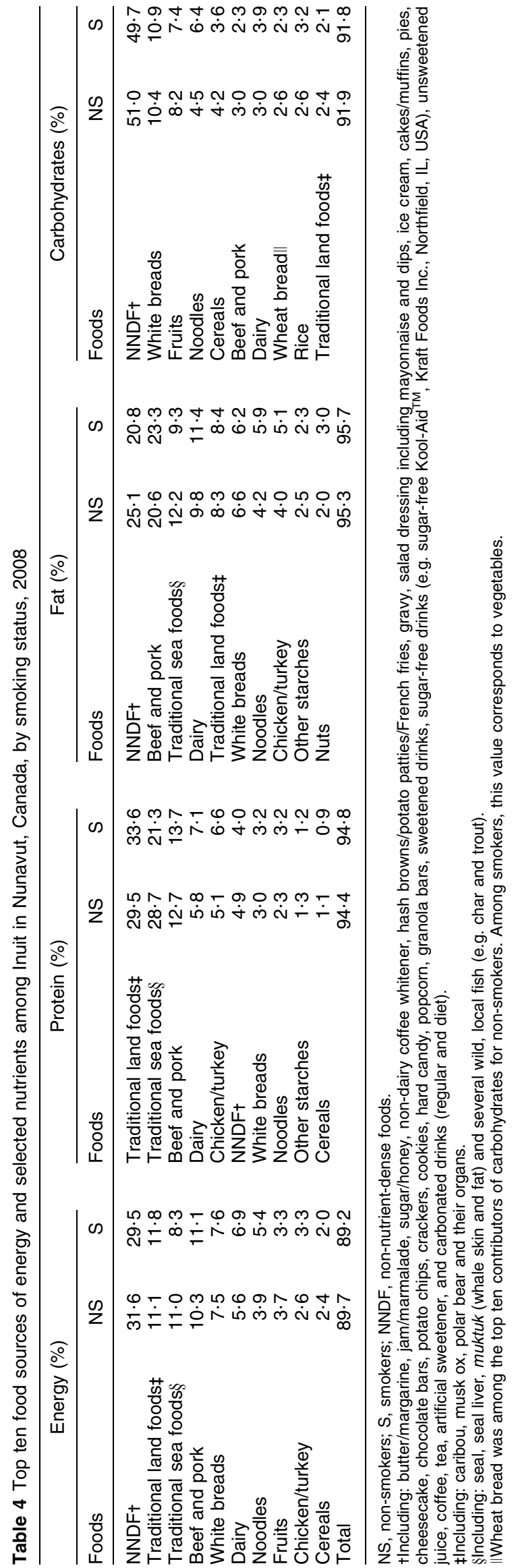

non-smokers, and fewer participants were below the DRI for these nutrients. Even so, between $20 \%$ and $36 \%$ of smokers were below the DRI for vitamin A, a nutrient which may have depleted serum concentration in smokers $^{(26,27)}$ and might be associated with decreased risk of anaemia ${ }^{(28,29)}$, which has been documented as highly prevalent among Inuit ${ }^{(30)}$.

A high proportion of smokers had nutrient profiles which might increase the risk of adverse cardiovascular outcomes. Average daily intake of Mg was above the DRI for male and female smokers; however, between $20 \%$ and $32 \%$ of all smokers were below recommended intakes. Mg is involved in a wide range of physiological mechanisms ${ }^{(31)}$ and low serum concentrations have been associated with $\mathrm{CVD}^{(31,32)}$ and diabetes ${ }^{(33)}$. Between $46 \%$ and $64 \%$ of smokers were below the recommended $\mathrm{K}$ intake. $\mathrm{K}$ is inversely related to the risk of hypertension and high $\mathrm{K}$ mitigates the blood-pressure-raising effects of $\mathrm{Na}^{(34)}$. This is notable among smokers as $\mathrm{Na}$ intakes were three to four times higher than the DRI. High Na, energy, saturated fat and cholesterol intakes combined with low $\mathrm{K}$ and $\mathrm{Mg}$ intakes might increase the risk of obesity, atherosclerosis, hypertension and CVD among smokers in this population ${ }^{(33,35)}$.

Differences in dietary quality between female smokers and non-smokers was evident in the lower intake density of several nutrients among smokers, many of which are consistent with the lower intake of traditional foods among this group ${ }^{(36)}$. These differences cannot be attributed to smoking alone, as female smokers were significantly younger than non-smokers and age has been correlated with traditional food intake ${ }^{(22,37)}$. Stratifying respondents by demographic factors beyond gender was outside the scope of the present study. Certainly a wide range of demographic factors contribute to smoking and dietary intake; however, the primary aim of the study was to characterize the dietary intake by smoking status, regardless of factors that might have contributed to a participant's smoking status. Considering dietary patterns, the high contribution of NNDF and low contribution of traditional food to energy intake was consistent with the ongoing nutritional transition in this population ${ }^{(18)}$. Traditional foods such as caribou, musk ox and marine mammals are rich in protein, polyunsaturated fats, vitamins $\mathrm{A}$ and $\mathrm{D}$, several $\mathrm{B}$ vitamins and $\mathrm{Fe}^{(36,38)}$. The lower dietary quality documented among female smokers reinforces previous recommendations ${ }^{(39)}$ of promoting nutrient-rich diets, primarily from traditional food sources.

The present study is not without limitations. Targeted participants for the study included the main food shopper/preparer of the household who are mostly women, thus there was a lower amount of male participants. This likely contributed to the lack of difference seen between male smokers and non-smokers and limits the generalizability of these results to the male population. 
The small overall sample size prohibited in-depth exploration and inferential statistical comparisons of dietary intake differences by level of smoking and behaviours that affect dietary intake, smoking and chronic disease risk, such as physical activity ${ }^{(40)}$ or alcohol consumption $^{(41-43)}$. Previous literature ${ }^{(44,45)}$ has discussed dietary intake differences based on socio-economic status (SES), including a study among this population ${ }^{(37)}$ which documented higher consumption frequency of traditional foods, fruits and vegetables among Inuit men and women with higher material style of life scores, a proxy for SES. Despite these limitations, the present study was able to provide a general overview of the dietary intake differences between Inuit smokers and non-smokers. Although it provided mixed results on the dietary adequacy varying by gender and smoking status, our study highlights nutrients affected by smoking and the potential increased risk for diet-related chronic diseases. Further exploration of the differences in dietary adequacy among Inuit by smoking status should consider disaggregating participants as only smokers, only alcohol consumers and participants who use both substances to further understand the effects of smoking and alcohol use on dietary adequacy.

\section{Conclusions}

Adult smokers in the present study consumed fewer nutrient-dense, traditional foods and had increased energy intake which likely contributed to fewer dietary inadequacies compared with non-smokers. Monitoring the dietary adequacy of smokers could prove useful for preventing diet- and smoking-related chronic diseases, especially in populations undergoing a nutrition transition and that experience an increased risk of CVD and aero-digestive cancers. The study highlights the need for comprehensive and community-based healthy lifestyle programmes that address the effects of food choice, dietary intake, physical activity and smoking on overall health. Further, studies exploring dietary adequacy of smokers may help to identify potential policy interventions to create supportive environments that enable individual behaviour change. In addition, a multidimensional approach to the reduction of risk for chronic disease in this population may be more effective in addressing clustered risk factors such as smoking and poor diet.

\section{Acknowledgements}

The project was supported by American Diabetes Association Clinical Research award 1-08-CR-57. All authors declare they have no conflicts of interest. S.E.R. interpreted the data and drafted the manuscript. C.R. participated in the acquisition and interpretation of the data. S.S. was responsible for the conception and design of the study. All authors critically reviewed and approved the final manuscript. The authors extend special thanks to Ms Eva Erber for performing the statistical analysis.

\section{References}

1. Inuit Tapiriit Kanatami (2009) Inuit \& cancer: fact sheets. http://www.itk.ca/publications/inuit-and-cancer-fact-sheets (accessed April 2010).

2. Heart and Stroke Foundation of Canada (2000) The Changing Face of Heart Disease and Stroke in Canada. http://www.statcan.gc.ca/pub/82f0076x/4227745-eng.pdf (accessed May 2010).

3. Nielson NH, Storm HH, Gaudette LA et al. (1996) Cancer in Circumpolar Inuit 1969-1988: a summary. Acta Oncol 35, 621-628

4. Bjerregaard P, Young TK, Dewailly E et al. (2004) Indigenous health in the Arctic: an overview of the circumpolar Inuit population. Scand J Public Health 32, 390-395.

5. Hopping BN, Mead E, Erber E et al. (2010) Dietary adequacy of Inuit in the Canadian Arctic. J Hum Nutr Diet 23, Suppl. 1, S27-S34.

6. Ockene IS \& Houston Miller N, for the American Heart Association Task Force on Risk Reduction (1997) Cigarette smoking, cardiovascular disease, and stroke: a statement for healthcare professionals from the American Heart Association. Circulation 96, 3243-3247.

7. Centers for Disease Control and Prevention, Office on Smoking and Health, National Center for Chronic Disease Prevention and Health Promotion (2009) Smoking and tobacco use: health effects of cigarette smoking. http:// www.cdc.gov/tobacco/data_statistics/fact_sheets/health_ effects/effects_cig_smoking/\#disparities (accessed June 2010).

8. Popkin BM (2002) An overview on the nutrition transition and its health implications: the Bellagio meeting. Public Health Nutr 5, 93-103.

9. Davis N, Katz S \& Wylie-Rosett J (2007) The effect of diet on endothelial function. Cardiol Rev 15, 62-66.

10. Powell JT (1998) Vascular damage from smoking: disease mechanisms at the arterial wall. Vasc Med 3, 21-28.

11. Erhardt L (2009) Cigarette smoking: an undertreated risk factor for cardiovascular disease. Atherosclerosis 205, 23-32.

12. Dallongeville J, Marécaux N, Fruchart JC et al. (1998) Cigarette smoking is associated with unhealthy patterns of nutrient intake: a meta-analysis. J Nutr 128, 1450-1457.

13. Northrop-Clewes CA \& Thurnham DI (2007) Monitoring micronutrients in cigarette smokers. Clin Cbim Acta 377, $14-38$.

14. Margetts BM \& Jackson AA (1993) Interactions between people's diet and their smoking habits: the dietary and nutritional survey of British adults. BMJ 307, 1381-1384.

15. Palaniappan U, Starkey LJ, O'Loughlin J et al. (2001) Fruit and vegetable consumption is lower and saturated fat intake is higher among Canadians reporting smoking. J Nutr 131, 1952-1958.

16. Tait H (2008) Aboriginal Peoples Survey, 2006: Inuit health and social conditions: supporting data tables. http:// www.statcan.gc.ca/bsolc/olc-cel/olc-cel?catno $=89-637$ XIE2008002\&lang=eng\#formatdisp (accessed April 2010).

17. Sharma S (2010) Assessing dietary intake in a population undergoing a rapid transition in diet and lifestyle: the Arctic Inuit in Nunavut, Canada. Br J Nutr 103, 749-759.

18. Sharma S (2010) Assessing diet and lifestyle in the Canadian Arctic Inuit and Inuivialuit to inform a nutrition and physical activity intervention programme. J Hum Nutr Diet 23, Suppl. 1, S5-S17. 
19. Pakseresht M \& Sharma S (2010) Validation of a quantitative food frequency questionnaire for Inuit population in Nunavut, Canada. J Hum Nutr Diet 23, Suppl. 1, S67-S74.

20. Health Canada, Nutrition Research Division (2010) Canadian Nutrient File 2007. http://www.hc-sc.gc.ca/fn-an/nutrition/ fiche-nutri-data/cnf_downloads-telechargement_fcen-eng.php (accessed February 2010).

21. Institute of Medicine of the National Academies (2005) Dietary Reference Intakes for Energy, Carbohydrates, Fiber, Fat, Fatty Acids, Cholesterol, Protein and Amino Acids. Washington, DC: The National Academies Press.

22. Kuhnlein HV, Receveur O, Soueida R et al. (2004) Arctic indigenous peoples experience the nutrition transition with changing dietary patterns and obesity. J Nutr 134, $1447-1453$.

23. Bjerregaard P \& Young KT (1998) Personal life style and health practices. In The Circumpolar Inuit - Health of a Population in Transition, pp. 206-213. Copenhagen: Munksgaard

24. Institute of Medicine of the National Academies (2003) Dietary Reference Intakes: Applications in Dietary Planning. http://www.nap.edu/openbook.php?record_id= 10609\&page $=\mathrm{R} 1 \#$ (accessed April 2010).

25. Schectman G, Byrd JC \& Hoffman R (1991) Ascorbic acid requirements for smokers: analysis of population survey. Am J Clin Nutr 53, 1466-1470.

26. Handelman GJ, Packer L \& Cross CL (1996) Destruction of tocopherols, carotenoids, and retinols in human plasma by cigarette smoke. Am J Clin Nutr 63, 559-565.

27. Alberg AJ (2002) The influence of cigarette smoke on circulating concentrations of antioxidant nutrients. Toxicology 180, 121-137.

28. Lynch SR (1997) Interaction of iron with other nutrients. Nutr Rev 55, 102-110.

29. Fishman SM, Christian P \& West KP (2000) The role of vitamins in the prevention and control of anaemia. Public Health Nutr 3, 125-150.

30. Jamieson JA \& Kuhnlein HV (2008) The paradox of anemia with high meat intake: a review of the multifactorial etiology of anemia in the Inuit of North America. Nutr Rev 66, 256-271.

31. Saris NEL, Mervaala E, Karpannen H et al. (2000) Magnesium: an update on physiological, clinical and analytical aspects. Clin Chim Acta 294, 1-26.

32. Touyz RM (2004) Magnesium in clinical medicine. Front Biosci 9, 1278-1293.
33. Simmons D, Joshi S \& Shaw J (2010) Hypomagnesaemia is associated with diabetes: not pre-diabetes, obesity or the metabolic syndrome. Diabetes Res Clin Pract 87, 261-266.

34. Adrogue HJ \& Madias NE (2007) Sodium and potassium in the pathogenesis of hypertension. $N$ Engl J Med 356, 1966-1978.

35. Appel LJ, for the American Society of Hypertension Working Group (2009) ASH position paper: dietary approaches to lower blood pressure. J Clin Hypertens (Greenwich) 11, 358-368.

36. Kuhnlein HV, Chan HM, Leggee D et al. (2002) Macronutrient, mineral and fatty acid composition of Canadian Arctic traditional foods. J Food Compost Anal 15, 545-566.

37. Hopping BN, Erber E, Mead E et al. (2010) Socioeconomic indicators and frequency of traditional food, junk food, and fruit and vegetable consumption amongst Inuit adults in the Canadian Arctic. J Hum Nutr Diet 23, Suppl. 1, S51-S58.

38. Kuhnlein HV, Barthet V, Farren A et al. (2006) Vitamins A, $\mathrm{D}$, and $\mathrm{E}$ in Canadian Arctic traditional food and adult diets. J Food Compost Anal 19, 495-506.

39. Kuhnlein HV \& Receveur O (2007) Local cultural animal food contributes high levels of nutrients for Arctic Canadian indigenous adults and children. J Nutr 137, 1110-1114.

40. Heath GW (2009) Physical activity transitions and chronic disease. Am J Lifestyle Med 3, issue 1, 27S-31S.

41. Piasecki TM, McCarthy DE, Fiore MC et al. (2008) Alcohol consumption, smoking urge, and the reinforcing effects of cigarettes: an ecological study. Psychol Addict Behav 22, 230-239.

42. Yeomans MR (2010) Alcohol, appetite and energy balance: is alcohol intake a risk factor for obesity? Physiol Behav 100, 82-89.

43. Rehm J, Room R, Graham K et al. (2003) The relationship of average volume of alcohol consumption and patterns of drinking to burden of disease: an overview. Addiction $\mathbf{9 8 ,}$ 1209-1228.

44. Stringhini S, Sabia S, Shipley M et al. (2010) Association of socioeconomic position with heath behaviors and mortality. JAMA 303, 1159-1166.

45. James PT, Nelson M, Ralph A et al. (1997) Socioeconomic determinants of health: the contribution of nutrition inequalities to health. BMJ 314, 1545-1549.

46. World Health Organization (2003) Diet, Nutrition and the Prevention of Chronic Diseases. Report of a Joint WHO/FAO Expert Consultation. WHO Technical Report Series no. 916, pp. 82-94. Geneva: WHO. 